\title{
Open heart mitral valve replacement using the Edwards-Sapien 3 for severe mitral annular calcification prevents left ventricular outflow tract obstruction
}

\author{
shohei morita ${ }^{1}$, Shinya Takahashi ${ }^{1}$, Mathieu Pernot $^{2}$, lionel leroux ${ }^{2}$, and Louis Labrousse ${ }^{2}$ \\ ${ }^{1}$ Hiroshima University Hospital \\ ${ }^{2}$ haut-leveque hospital
}

July 8, 2020

\begin{abstract}
We can safely treat mitral valve disease with mitral annular calcification using Sapien 3 valve through the incision of the right side of the left atrium under direct visualization preventing atrio-ventricular disruption and left ventricular outflow tract obstruction.
\end{abstract}

\section{1 | INTRODUCTION}

Open heart surgical use of a transcatheter aortic valve implantation (TAVI) prosthesis for mitral stenosis (MS) with severe MAC appears to decrease the risk of atrio-ventricular disruption following decalcification and circumflex artery injury, and simplifies the procedure ${ }^{1,2}$. And this procedure has no risk of systolic anterior motion of the anterior mitral valve leaflet (AML) compared to percutaneous transcatheter mitral valve implantation (TMVI), because we can resect the AML in open surgery. A potential problem with this procedure that should be addressed is a left ventricular outflow tract (LVOT) obstruction by the prosthesis, especially by the felt strip at the prosthesis ${ }^{1}$. This problem may especially occur when pre-operative LVOT is reduced by myocardial hypertrophy, which is usually observed when an associated aortic stenosis (AS) is present.

\section{2 | CASE DESCRIPTION}

A 74-year-old man presented with New York Heart Association class II due to AS and MS.

Echocardiography showed severe MS with a mean pressure gradient (PG) of $12 \mathrm{mmHg}$ and a mitral valve $(\mathrm{MV})$ area of $0.99 \mathrm{~cm}^{2}$ (Figure. 1A). The left ventricle (LV) was hypertrophic and the LV ejection fraction was $70 \%$. AS was also severe and the aortic valve (AV) area was $0.74 \mathrm{~cm}^{2}$. MAC was severe as shown on computed tomography (CT) (Figure. 1B). The pre-operative aortomitral angle was 110(Figure. 1C), so the risk of LVOT obstruction was high ${ }^{3}$. The width of the pre-operative LVOT in the direction of placing the TAVI prosthesis was $20.3 \mathrm{~mm}$ (Figure. 1D). The extra space after performing TMVI becomes neo-LVOT. We can estimate post-procedural LVOT obstruction taking measurement of neo-LVOT at the baseline CT scan.

After a median sternotomy on cardiopulmonary bypass with cardioplegic cardiac arrest, the calcified AV was resected through an aortotomy. The aortic annulus was small and a 19-mm AVALUS valve sizer (Medtronic, Minneapolis, MN, USA) was suitable. The left atrium was opened parallel to the interatrial groove. We resected the A2 valve to prevent anterior MV protrusion in the LVOT and secondary LVOT obstruction. We sewed anchor sutures around the mitral annulus (2-0 coated braided polyester sutures with pledgets) in 
places where needles exhibited no resistance around or across calcifications. These sutures were used to fix the final position of the TAVI prosthesis once inflated. We measured the mitral annulus using valve sizers of mitral mechanical valve and we chose a valve that was exactly the same size as the annulus. As previously described $^{2}$, after washing a 26-mm Sapien-Edwards AV (Edwards Lifesciences, Inc., Irvine, CA, USA), felt strip sewn to the Sapien to cover the polyethylene terephthalate portion (Figure. 2A). The prosthesis was mounted upside down on the short delivery catheter. A Safari XS (Boston Scientific, Natick, MA, USA) was passed into the catheter to avoid injury of the LV. The balloon was inflated, the valve was deployed under direct visualization (Figure. 2B). During that step, it was necessary to assess the position of the A1 and A2 parts of the mitral annulus on the felt strip to reduce the amount of covered stent into the LVOT. Usually the width of the felt strip is approximately $1 \mathrm{~cm}$ and a residual 5-6 $\mathrm{mm}$ width in the atrial side is likely to avoid LVOT obstruction. Then, the valve was fixed in its position by passing the previous anchor sutures across the bottom of the Sapien and tied with the Corknot device (LSI Solutions, Victor, NY, USA) to prevent valve migration. These sutures, in our opinion, also reduced paravalvular leaks (PVL) (Figure. 2C). After we closed the left atrium, we replaced the AV with a 19-mm AVALUS (Medtronic). Visualization through the aortic annulus confirmed that only a limited part of the felt strip was positioned into the LVOT, thus obstruction of the LVOT was not likely to occur (Figure. 2D). After we closed the aorta, the cross-clamp was removed after $96 \mathrm{~min}$. While weaning from cardiopulmonary bypass, we temporarily stopped the bypass flow and checked the PG between the LV $(109 / 12 \mathrm{mmHg})$ (needle through the ventricular wall) and the ascending aorta $(94 / 56 \mathrm{mmHg})$. Because the direct pressure is higher than the pressure measured by echocardiography, and the mean gradient measured by echocardiography after AV replacement using AVALUS is between 10 and $15 \mathrm{mmHg}$, this maximum PG was acceptable ${ }^{4}$ Transthoracic echocardiography 3 month post-operatively showed no PVL at the mitral prosthesis and confirmed no significant LVOT obstruction.

\section{3 | DISCUSSION}

Mayra et al. ${ }^{1}$ reported a multicenter global registry involving the acceptable outcomes of early experience with TMVI in patients with $\mathrm{MAC}^{1}$. However, the 30-day and 1-year all-cause mortality rates were $25 \%$ and $53.7 \%$, respectively. It was concluded that LVOT obstruction is the most important and independent predictor of 30-day and 1-year mortality ${ }^{5}$.

Although cross-clamping and cardioplegia were required, this technique allows precise assessment of positioning along A1 and A2 of the covered part of the TAVI and resection of A2. Indeed, these two points are advantages in avoiding LVOT obstruction compared to percutaneous TMVI. John-Peder et $a l .{ }^{6}$ reported a case of LVOT obstruction after trans-apical TMVI, and the patient needed for secondary surgery to resect the A1-A2-A3 parts of the MV.

In percutaneous TMVI, a laceration of the AML may be performed to prevent LVOT obstruction (LAMPOON procedure). Vasilis et al. ${ }^{7}$ reported five successful cases of patients at risk for LVOT obstruction who underwent the LAMPOON procedure and percutaneous TMVI using the Sapien 3; however this technique does not appear to be appropriate for calcified AML.

Patients with AS and mitral disease associated with MAC can be treated with associated classical AV replacement and trans-atrial open heart TMVI. During balloon inflation, this approach allows a very good assessment of the width of the covered TAVI prosthesis positioning into the LVOT and might play a major role in reducing the risk of LVOT obstruction; however, more experience is needed to confirm this preliminary result.

\section{3 | CONCLUSION}

Open heart mitral valve replacement using the Edwards-Sapien 3 for severe mitral annular calcification (MAC) is a safe procedure, because we don't need to decalcify the MAC. And also, we can resect the anterior leaflet to prevent left ventricular outflow tract obstruction and we can deploy it under direct visualization.

Conflicts of interest

Dr L. Leroux is a proctor for Edwards Lifescience. 
The other authors declare that there are no conflicts of interest.

Acknowledgments

None

Ethics statement

The authors confirm that written consent for submission and publication of this case report.

Author contribution

SM, ST, MP, LL and LL: made major contributions to the writing of this manuscript. All authors read and approved the final manuscript.

\section{REFERENCES}

1. Guerrero M, Dvir D, Himbert D, Urena M, Eleid M, Wang DD, et al. Transcatheter mitral valve replacement in native mitral valve disease with severe mitral annular calcification: Results from the first multicenter global registry. J Am Coll Cardiol Intv 2016;9:1361-71.

2. Lee R, Fukuhara S, George I, Borger MA. Mitral valve replacement with a transcatheter valve in the setting of severe mitral annular calcification. J Thorac Cardiovasc Surg 2016;151:e47-9.

3. Varghese R, Itagaki S, Anyanwu AC, Trigo P, Fischer G, Adams DH. Predicting systolic anterior motion after mitral valve reconstruction: using intraoperative transoesophageal echocardiography to identify those at greatest risk. Eur J Cardiothorac Surg 2014;45:132-8.

4. Klautz R J.M., Kappetein A.P, Lange R, Dagenais F, Labrousse L, Bapat V. Safety, effectiveness and haemodynamic performance of a new stented aortic valve bioprosthesis. Eur J of Cardiothorac Surg 2017;52:425-431.

5. Guerrero M, Urena M, Himbert D, Wang DD, Eleid M, Kodali S, et al. 1-year outcomes of transcatheter mitral valve replacement in patients with severe mitral annular calcification. J Am Coll Cardiol 2018;71:1841-53.

6. Kvitting JPE, Nielsen NE, Vanhanen I, Baranowski J. Surgical management of outflow tract obstruction after transapical mitral valve implantation. J Card Surg 2018;33:545-547.

7. Babaliaros VC, Greenbaum AB, Khan JM, Rogers T, Wang DD, Eng MH. Intentional percutaneous laceration of the anterior mitral leaflet to prevent outflow obstruction during transcatheter mitral valve replacement. JACC Cardiovasc Interv 2017;10:798-809.

Legends

FIGURE 1 A, Pre-operative transesophageal echocardiography showed calcified MV and MAC with turbulent flow at the MV in systole. B, Pre-operative CT showed MAC. C, Aortomitral angle was 110. D, Pre-operative LVOT. The measurement is parallel to the TAVI prosthesis.

FIGURE 2 A, Felt strip sewn to the Sapien to cover the polyethylene terephthalate portion. B, Valve implantation. C, After the valve implantation, anchor sutures were sewn to the felt strip and tied thereafter. D, Visualization via the aortotomy enabled us to view the Sapien at the mitral position and there was no obstruction at the LVOT. 

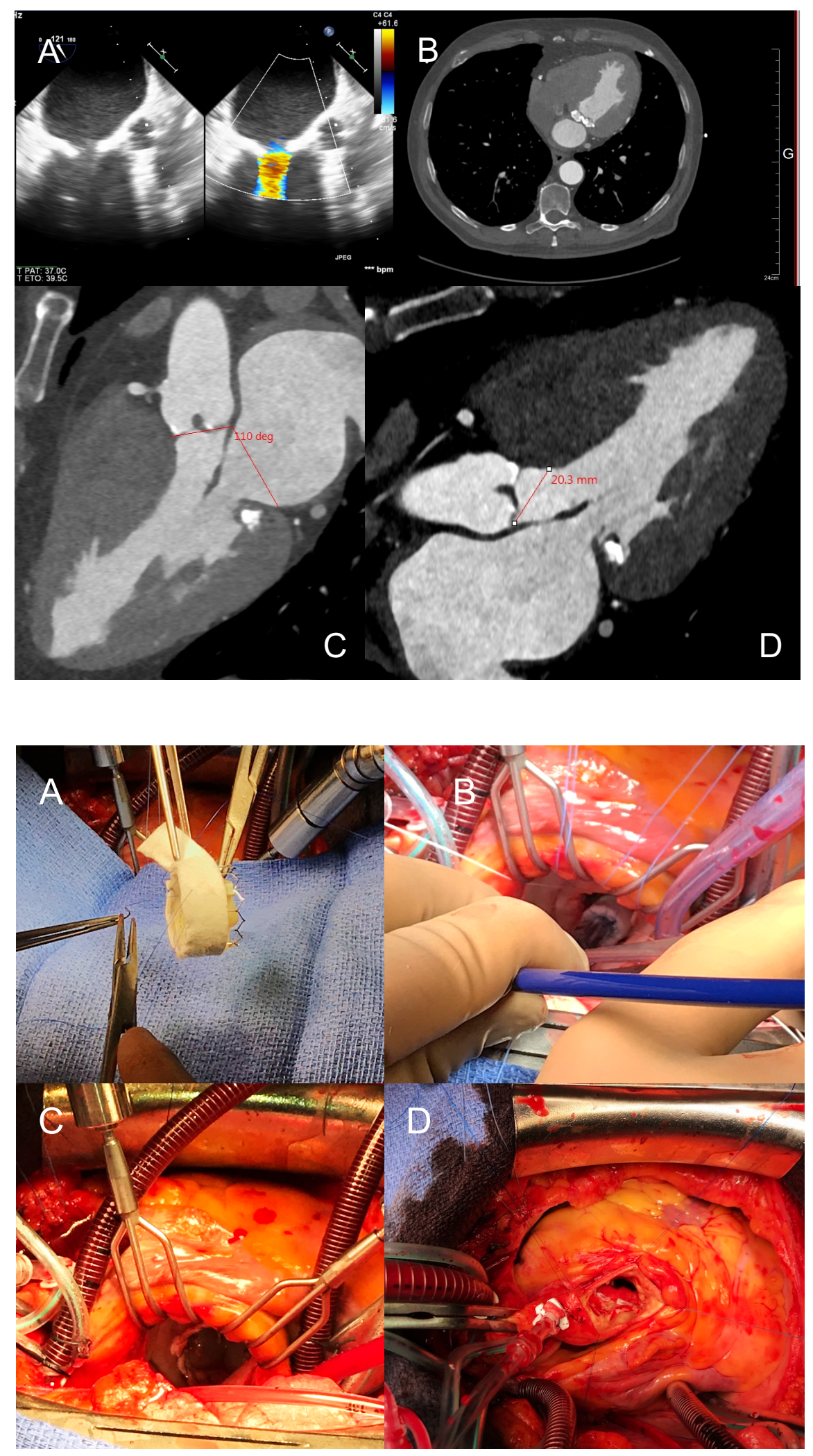